\title{
ESTUDIO TRADUCTOLÓGICO DE LOS VERBOS SINTAGMÁTICOS DEL ITALIANO AL CASTELLANO. EL CASO DE LESSICO FAMIGLIARE ${ }^{1}$
}

\author{
Andrea Artusi \\ andrea.artusi@uv.es \\ Universitat de València - IULMA
}

\section{Resumen}

Los verbos sintagmáticos (VS) constituyen un fenómeno lingüístico muy productivo en la lengua italiana, en particular en los intercambios dialógicos más informales y espontáneos. Ante la parca productividad de dichos constructos en español, destacamos los recursos lingüísticos que se suelen emplear con más frecuencia en este idioma al traducir los VS italianos. Para ello, analizamos un corpus paralelo, basado en la obra Lessico famigliare de Natalia Ginzburg (1963) y su traducción al español. Tras detallar los VS que figuran en el texto origen, adoptamos un enfoque de tipo cognitivo para dilucidar las posibles causas de aparición de los patrones lingüísticos destacados en la traducción. Por último, tras señalar algunos errores de traducción que se hallan en el texto meta y los motivos por los que se producen, planteamos que los VS han de considerarse como problemas de traducción.

\begin{abstract}
"A corpus-based translation study on Italian verb-particle constructions translated into Spanish. The case of Lessico famigliare"

Verb-particle constructions (VPCs) are considered to be a very productive linguistic phenomenon within the Italian language, especially in spontaneous and informal dialogues. On the contrary, in the Spanish language the productivity of such constructions appears to be low. In order to figure out which linguistic patterns seem to
\end{abstract}

1. Artículo elaborado en el marco del Proyecto de investigación concedido por el Ministerio de Economía y Competitividad. Referencia FFI2011-24712, Análisis léxico y discursivo de corpus paralelos y comparables (español, inglés y francés) de páginas electrónicas de promoción turística. 2011-2014. 
be more frequent while translating Italian verb-particle constructions into Spanish, a parallel corpus will be analysed. The corpus consists of the Italian novel Lessico famigliare (by Natalia Ginzburg, 1963) and its translation into Spanish. First, a list of the VPCs contained in the source text will be provided. Secondly, we will show which linguistic patterns are used in the translation, elucidating the reasons why they tend to appear, by means of a cognitive approach. Finally, some translation errors will be highlighted. The possible causes that resulted in such errors will lead us to allege that VPCs should be considered as translation problems.

Key words: Orality. Translation. Verb-Particle Constructions. Translation Techniques. Translation Problem.

Palabras clave: Oralidad. Traducción. Verbos sintagmáticos. Técnicas de traducción. Problema de traducción.

Manuscript received on April 12, 2015 and accepted for publication on September $18,2015$. 


\section{Introducción}

Hasta tiempos relativamente recientes, en el seno de las investigaciones de lingüística italiana, el fenómeno de los verbos sintagmáticos (de ahora en adelante VS) ha sido sorprendentemente ignorado por parte de los estudiosos, a pesar de su elevada productividad y frecuencia de uso tanto en los textos escritos como en las interacciones orales de la comunidad de hablantes nativos de italiano. Una consecuencia directa de ello es que, en la actualidad, ni las principales gramáticas de italiano ofrecen análisis exhaustivos de dichas manifestaciones lingüísticas (Simone 1997: 157), ni las fuentes lexicográficas monolingües y bilingües parecen otorgarles un tratamiento homogéneo, coherente y sistemático (Calvo 2010: 375).

Por otra parte, en español, los VS presentan una productividad muy baja y -a diferencia de lo que ocurre en italiano- no se consideran una categoría verbal que destaque frente a otras, ya que no cuentan con un comportamiento sintáctico, semántico y fonético reconocible (Calvo 2008: 60-63).

Así pues, ante la falta de una correspondencia entre el italiano y el español en lo que se refiere a estas estructuras lingüísticas, el análisis de corpus paralelos puede servirnos de punto de partida para observar de qué manera, al traducirse los constructos sintagmáticos verbales italianos, el sistema lingüístico del español hace frente a semejante "vacío" sintáctico.

Tras unas rápidas pinceladas para recordar las características sintácticas y semánticas propias de los VS, procederemos a analizar la traducción, del italiano al español, de las construcciones sintagmáticas verbales que figuran en el corpus objeto de análisis: la obra Lessico famigliare, de Natalia Ginzburg (1963).

Tal y como observaremos, debido al carácter marcado desde el punto de vista diafásico y diamésico² de los VS, partimos de la hipótesis inicial según la

2. Este término, poco usual en la lingüística española, pero frecuente en la italiana, fue acunado por Alberto Mioni (1983: 508-510), y hace referencia a la variación ligada al canal de comunicación; en nuestro caso, se refiere al canal oral, es decir, todo lo relacionado con la oralidad. 
cual el corpus seleccionado constituye un texto ideal para la aparición de los constructos en cuestión, pues se trata de una novela que guarda una relación muy estrecha con la oralidad.

Tras confirmar dicha hipótesis, al haber obtenido un extenso listado de VS que figuran en la obra, procederemos a subrayar cómo se comporta el castellano ante la traducción de un estímulo lingüístico dado (VS italiano), y aprovecharemos la ocasión para destacar algunas diferencias de carácter tipológico entre el sistema lingüístico italiano y el español, atendiendo a un enfoque de tipo cognitivo.

A continuación, procederemos a detallar algunos errores de traducción de distinta naturaleza, mediante una postura traductológica de carácter funcional, con el objeto de señalar las posibles causas de su aparición, y mostrar, de ese modo, su repercusión e incidencia. Todo ello nos llevará a postular que, al menos para la traductora de la obra, los VS italianos constituyen a todos los efectos un problema de traducción.

Las observaciones que expondremos en esta aportación no pretenden en absoluto tener un alcance general en lo que se refiere a la traducción de los VS del italiano al español, sino que, como es lógico, se limitan única y exclusivamente al corpus analizado.

\subsection{Definición y características de los VS italianos}

Si bien la existencia de los VS ya se había observado con anterioridad (Carrera 1984: 277ss.; Schwarze 1985: 355-371), la primera aportación que, a nuestro parecer, arrojó más luz sobre la cuestión y, a la vez, contribuyó a estimular la producción científica al respecto, es la de Simone, quien define los VS como:

sintagmi formati da una testa verbale e da un complemento costituito da una 'particella' (originariamente un avverbio), uniti da una coesione sintattica di grado elevato al punto che non si può commutare il VS intero con una sola delle sue parti. Si tratta quindi di costruzioni del tipo di fare fuori, venire meno, buttare giù. (Simone 1997: 156-157)

Desde el punto de vista sintáctico, varios autores han observado una serie de características propias de los VS, que nos permiten diferenciar estos constructos de la simple asociación V+Adv. y V+SP, ya que, en estos últimos, el adverbio y el sintagma preposicional parecen ser autónomos y libres de vínculos frente al verbo, a diferencia de lo que sucede con los VS, en los que el grado de cohesión entre los elementos que los componen -un núcleo verbal (V) y una partícula (P) - es muy elevado. De hecho, tal y como observa Masini (2012: 
196-200), al resumir lo que ella misma y otros investigadores han señalado al respecto ${ }^{3}$ :

- En las secuencias V+SP y V+SAdv. el orden de los constituyentes puede variar y se acepta la inserción de material argumental entre las partes que las componen ( $1 \mathrm{a}, 1 \mathrm{~b}, 1 \mathrm{c}, 1 \mathrm{~d}$ ); en cambio, en los VS transitivos, el V y la $\mathrm{P}$, en la mayoría de las ocasiones ${ }^{4}$, pueden estar separados exclusivamente por elementos de carácter no argumental (2c), ya que, de lo contrario, darían lugar a oraciones agramaticales (2b):

(1) a. Luca ha lavato la macchia con il sapone

b. Luca ha lavato con il sapone la macchia

c. Luca ha lavato con accanimento la macchia

d. Luca ha lavato la macchia con accanimento

(2) a. Luca ha lavato via la macchia

b. *Luca ha lavato la macchiavia

c. Luca ha lavato subitovia la macchia

- Ante un V+SP o V+SAdv. se pueden llevar a cabo dislocaciones (3b) y topicalizaciones (3c); por otra parte, los VS (en particular, los más lexicalizados) no permiten la dislocación a la izquierda de la P (4b), ni la topicalización (4c):

(3) a. È andato là dentro

b. È là dentro che è andato

c. Là dentro è andato

(4) a. Abbiamo messo su il caffè

b. * È su che abbiamo messo il caffè

c. *Su abbiamo messo il caffè

- A diferencia de lo que ocurre en V+SP y V+Adv. (5a,5b,5c), las P que dan lugar a los VS se muestran reacias a la coordinación $(6 a, 6 b)$ :

3. Reproducimos aquí los ejemplos proporcionados por esta lingüista.

4. En un estudio anterior, Masini apunta que, en algunos casos puntuales de VS, se observa la inserción entre V y P de material argumental, debido a razones de tipo pragmático. A este respecto, Masini concluye "non possiamo dire che la costruzione discontinua sia in alternanza con la costruzione continua come in inglese. Possiamo tuttavia dire che la costruzione discontinua è esclusa in determinati contesti pragmatici, ovvero in contesti in cui l'oggetto è focalizzato" (Masini 2008: 100). 
(5) a. Marco sta dietro a Giovanni edavanti a Sandra

b. Marco sta dietro a Giovanni e Maria davanti a Sandra

c. Marco ha pulito la casa dentro e fuori

(6) a. ?Sara ha messo dentro la bici e fuori la spazzatura

b. " Sara ha portato fuori le bici e Luca dentro la spesa

- Si por un lado la combinación V+SP acepta dos tipos de nominalización (infinitivo nominal y sustantivo deverbal) (7b, 7c), la nominalización de los VS es posible solo bajo la forma de infinitivo nominal $(8 \mathrm{~b}, 8 \mathrm{c})$ :

(7) a. La gente corre vicino alla pista

b. Il correre della gente vicino alla pista

c. La corsa della gente vicino alla pista

(8) a. Gianni è corso via subito dopo la partita

b. * La corsa via di Gianni subito dopo la partita

c. Il correre via di Gianni subito dopo la partita

Por último, tal y como apunta Calvo (2008: 51-52), dado un VS, no parece posible separar la $\mathrm{P}$ del núcleo verbal mediante el empleo de interrogaciones (9b):

(9) a. Il cliente è stato cacciato via in malo modo

b. Dove è stato cacciato il cliente in malo modo? *Via

Las consideraciones que hemos traído a colación nos han servido de pruebas sintácticas con el objeto de determinar qué constructos del corpus analizado pertenecen a la categoría de los VS y seleccionarlos de forma rigurosa ${ }^{5}$.

En cuanto a las características semánticas de los VS, dentro de la categoría de estos constructos verbales se observan diferentes subgrupos: el primero, en el cual la P que acompaña al V no hace más que reiterar o intensificar lo que ya está expresado por el verbo (uscire fuori); el segundo, en el cual la $\mathrm{P}$ al asociarse normalmente a un verbo de movimiento no marcado, determina la dirección

5. En la bibliografía se han señalado diferentes tipos de P que pueden participar en la formación de numerosas clases diferentes de VS: locativa, deíctica, temporal, de manera y de cantidad (Masini 2012: 195-196). En nuestra investigación hemos estudiado única y exclusivamente los VS que contienen P locativas, pues constituyen el subgrupo de VS más representativo. 
del mismo (metter giù, tirare su, saltare dentro) ${ }^{6}$; el tercero, en el que la semántica del constructo verbal no es de tipo composicional, pues no es deducible de la suma del significado de sus componentes (como ocurre en fare fuori, que, por ejemplo, puede significar 'eliminar' o'matar') (Simone 1997: 162) ${ }^{7}$.

Por último, se ha demostrado que los VS del italiano moderno se caracterizan por una notable polisemia, es decir, un mismo VS tiende a adquirir múltiples significados, dependiendo del contexto en el que figure (Ježek \& Strik Lievers 2010:450).

\subsection{Los VS italianos en la oralidad}

Tal y como hemos señalado con anterioridad, los estudios realizados hasta la fecha han destacado que los VS italianos -considerados en su conjuntosuelen presentar, por lo general, un marcado carácter desde el punto de vista diafásico y diamésico.

De hecho, en un estudio (Antelmi 2002: 112) basado en corpus de textos tanto escritos (artículos periodísticos de ámbito económico y deportivo) como hablados (transcripciones de programas de televisión y conversaciones espontáneas) se hizo patente que existe una frecuencia de uso mayor de VS en los textos orales con respecto a los escritos periodísticos, siendo su empleo particularmente limitado en los artículos de temática económica. En definitiva, se demostró que cuanto mayor sea el carácter espontáneo e informal de un texto, mayor suele ser el empleo de complejos sintagmáticos verbales.

Asimismo, gracias a un estudio posterior, los resultados de un análisis sobre el corpus de italiano hablado LIP confirmaron que en los intercambios más informales el empleo de VS resulta notablemente mayor, sobre todo en los intercambios dialógicos en los que los interlocutores toman la palabra de forma libre (Iacobini 2008: 117).

Dado que nuestro objetivo consistía en comparar el italiano y el español, y más concretamente, estudiar cómo se traducen los VS, nos pareció que lo más lógico era analizar su presencia en textos escritos y en sus correspondientes

6. En lo que se refiere al segundo subgrupo, Venier (1996: 152) lleva a cabo una distinción entre, por un lado, aquellos VS en los que la $\mathrm{P}$ especifica la dirección del movimiento, a pesar de que esta ya esté expresada parcialmente porel V (gettare giù), y, por otro, los VS en los que la dirección del movimiento es expresada únicamente por P (tirare fuori).

7. En cuanto al tercer subgrupo, se observan usos de VS de tipo metafórico, que en la mayoría de los casos son el resultado de un proceso de lexicalización de VS, cuya P tiene valor locativo. La semántica de este tipo de VS puede ser en mayor o menor medida transparente, dependiendo de la relación que estos guardan con sus correspondientes VS de procedencia (Masini 2005: 154-156; Masini 2012: 201). 
traducciones ${ }^{8}$. Ahora bien, puesto que, como ya hemos afirmado, los VS se dan sobre todo en contextos informales y dialógicos, hemos escogido un texto literario con un elevado índice de estas características.

\section{El corpus objeto de análisis: Lessico famigliare}

Aunque sin lugar a dudas la novela objeto de análisis no necesita ningún tipo de presentación, al tratarse de una de las obras más importantes ${ }^{9}$ de la literatura italiana del siglo XX, consideramos oportuno recordar que Lessico famigliare narra la historia, ambientada principalmente en Turín entre principios de los años treinta y principios de los cincuenta del siglo pasado, de una familia judía y antifascista. La familia Levi no es fruto de la imaginación de Natalia Ginzburg, sino que constituye el núcleo familiar en el que la autora ${ }^{10}$ nació y se crió. A través de la memoria, Natalia recupera expresiones, exclamaciones y frases hechas que en su familia se solían utilizar muy a menudo y que acaban constituyendo los pilares de su unidad e identidad familiar. Además de abordar temas de sumo interés desde el punto de vista histórico y sociológico ${ }^{11}$, la obra destaca frente a las de su época por el reflejo de la oralidad familiar que ofrece al lector.

A lo largo de la obra se observa el empleo recurrente del discurso directo, una modalidad discursiva cuya inmediatez y expresividad parece reproducir de manera muy fiel los parlamentos que la autora oyó de pequeña entre las paredes de su casa. Sin embargo, se trata de una representación de las palabras del hogar de los Levi que difiere en mayor medida de la "original", ya que, a diferencia de lo que se podría pensar a primera vista, la enunciación de cada personaje no es independiente y autónoma frente a las de los demás, sino que

8. Desde el punto de vista metodológico, decidimos no basarnos en la observación de nuestras capacidades lingüísticas en calidad de hablantes nativos de italiano (L1) y de español como lengua extranjera (L2), sino que, por el contrario, optamos por estudiar datos externos, de tipo contrastivo. El empleo de un texto paralelo (traducción) es, a nuestra manera de ver, la única forma de poder hallar equivalentes de VS. Varios autores coinciden en la validez del análisis de traducciones con el objeto de llevar a cabo estudios contrastivos (Wandruszka 1969; Wandruszka 1971; Hartmann 1980; Valero 1995; Calvo 2005).

9. En el año de su publicación la obra fue galardonada con uno de los más prestigiosos reconocimientos literarios italianos, el Premio Strega. Además, no ha dejado nunca de editarse y se ha traducido a numerosos idiomas.

10. El verdadero apellido de la autora es Levi. En la edad adulta pasará a apellidarse Ginzburg tras el matrimonio con Leone Ginzburg, de acuerdo con la costumbre italiana de cambiar el apellido de la mujer por el de su marido.

11. Para un análisis detallado de los temas que se hallan en la obra, así como de su génesis, estructura y modelos literarios, véase Magrini (1996: 771-810). 
su conjunto da lugar a una estructura unitaria de nivel superior, esto es, a la enunciación de la autora (Barani 1990:148). En otras palabras, la autora se sirve de la memoria como principio organizativo para crear, en última instancia, un trabajo que ha de leerse como una novela basada en el recuerdo, en sus aportaciones y omisiones.

En cuanto a las características del estilo del Lessico, las intervenciones en estilo directo que figuran principalmente en la obra son las de los padres de Natalia. A pesar de que la mayoría de la novela esté redactada en lengua italiana, en sus páginas se aprecian también numerosas influencias dialectales.

Además, se trata de una lengua marcada desde el punto de vista diamésico, ya que presenta numerosas características de la oralidad: en ella no solo figuran estructuras agramaticales, sino que también se tratan temas y argumentos banales, típicos de las conversaciones cotidianas. Pese a ello, cabe suponer que las características del lenguaje utilizado no responden a una variedad diastrática baja, ya que los hablantes que participan en gran parte de los diálogos son cultos (Barani 1990:150).

Otro rasgo interesante que cabe mencionar es el carácter polifónico que presentan algunos discursos directos: en ellos, en efecto, el personaje que habla cita en ocasiones las palabras de otro, por razones muy diversas entre sí, que pueden variar según la intencionalidad de su acto de habla (burlarse de la persona a la que se cita, reconocer su autoridad, ser irónico o polémico frente a esa persona, etc.). En lo que respecta a los apartados de la obra en los que se utiliza el estilo indirecto, diremos que en este caso también se observan ejemplos de polifonía, en los que la voz del narrador acaba confundiéndose con la del personaje del que se está hablando, por adquirir el lenguaje los rasgos propios del idiolecto de este último. Dicha polifonía, junto con las expresiones idiolectales y dialectales de algunos personajes y la marcación hacia lo oral en el eje diamésico de la obra, suponen, en muchas ocasiones, verdaderos problemas de traducción, esto es, dificultades objetivas a las que se enfrenta el traductor a la hora de desempeñar su labor (Hurtado 2001: 286).

Ahora bien, a pesar de que -por las razones que acabamos de exponersería muy interesante desarrollar un trabajo de análisis traductológico de la novela en su conjunto, debido a las limitaciones de nuestra investigación y del espacio del que aquí disponemos, nos ceñiremos al estudio de la traducción de los VS que figuran en el texto origen (TO).

Antes de proceder a ello, en cuanto al texto meta (TM) de nuestro estudio traductológico, cabe señalar que la traducción al castellano de Lessico famigliare fue publicada por primera vez en 1989 por la editorial Trieste, y fue llevada a cabo por Mercedes Corral, una profesional que en la actualidad 
cuenta con treinta años de experiencia en el sector de la traducción literaria. A lo largo de su carrera, Corral se ha ocupado de la traducción al español de obras tanto italianas como francesas. Además, dirigió de 2005 a 2012 la Casa del Traductor ("Centro Hispánico de Traducción Literaria") de Tarazona.

\section{VS que figuran en el corpus}

Tras la labor de extracción de los VS que se hallan en la obra original italiana, podemos afirmar que en Lessico famigliare figura un número relativamente elevado de constructos sintagmáticos verbales, lo que demuestra que dicho fenómeno verbal goza de un buen nivel de productividad, incluso en un corpus limitado como el que aquí se analiza ${ }^{12}$.

Hallamos un total de 124 ejemplos de VS en términos de tokens. En la tabla que facilitamos a continuación se señalan los types relativos a las partículas (P), a los núcleos verbales (V) y a las estructuras sintagmáticas verbales (VS) que se hallan en el corpus, así como su frecuencia (tokens) entre paréntesis.

\begin{tabular}{|c|c|l|}
\hline & Types & \multicolumn{1}{c|}{ Tokens por type } \\
\hline $\mathrm{P}$ & 14 & $\begin{array}{l}\text { via (41); giù (16); fuori (12); su (12); dentro, dietro (11); indietro (5); } \\
\text { avanti, attorno (4); intorno, davanti, incontro (2); accanto, senza (1). }\end{array}$ \\
\hline $\mathrm{V}$ & 38 & $\begin{array}{l}\text { andare, buttare (14); mettere (13); tirare (10); essere (7); andarsene, } \\
\text { portare (6); mandare, portarsi (5); venire, tirarsi (4); guardarsi (3); } \\
\text { stare, cacciare, spazzare, buttarsi, passare (2); rivolere, sgusciare, uscire, } \\
\text { schiodare, entrare, riportare, trascinare, fuggire, ritornare, tenere, tro- } \\
\text { varsi, rimanere, scrutare, circolare, tagliare, venirsene, ricacciare, } \\
\text { vedersi, levare, starci, dare (1). }\end{array}$ \\
\hline VS 64 & $\begin{array}{l}\text { buttare via (10); mettere su, essere dentro (7); portare via, tirare fuori } \\
\text { (5); tirarsi dietro, andare giù, andarsene via, andare dietro (4); man- } \\
\text { dare via, andare via, portarsi dietro, buttare giù (3); venire giù, cacciare } \\
\text { via, guardarsi attorno, tirare su, spazzare via, buttarsi giù, scappare via, } \\
\text { mettere fuori, mettere dentro, andarsene giù, portarsi via (2); andare } \\
\text { su, tirare avanti, rivolere indietro, sgusciare via, andare avanti, uscire } \\
\text { fuori, guardarsi intorno, stare attorno, schiodare fuori, tirare giù, entrare } \\
\text { dentro, riportare indietro, trascinare via, fuggire via, ritornare indietro, } \\
\text { tenere su, andare fuori, trovarsi davanti, passare accanto, venire incontro, } \\
\text { mandare incontro, rimanere dentro, essere senza, venire fuori, scrutare } \\
\text { attorno, circolare attorno, passare giù, tagliare fuori, venirsene via, ricac- } \\
\text { ciare indietro, buttare indietro, vedersi davanti, mettere avanti, levare via, } \\
\text { tirare via, mandare avanti, starci su, portare giù, mettere via, dare via (1). }\end{array}$ \\
\hline
\end{tabular}

Tabla 1. Types y tokens de V, P y VS

12. Asciende a unas 64000 palabras. 
La asociación de tan solo 14 partículas y 38 núcleos verbales en términos de types conlleva la formación de $64 \mathrm{VS}$, un dato que valoramos como particularmente elevado si consideramos las dimensiones limitadas del corpus, lo que parece confirmar una vez más la productividad de esta categoría verbal. En cuanto a los componentes de los VS, diremos que la mitad de las partículas presenta un número de tokens muy elevado, que de 5 apariciones llega a ascender a 41 en el caso de via. Además, cabe señalar que, frente a un número exiguo de $\mathrm{V}$ y de $\mathrm{P}$ que presentan una frecuencia muy elevada en cuanto a sus ocurrencias textuales (tokens), la mayoría de partículas y núcleos verbales da lugar solo a una estructura sintagmática o poco más. Por otra parte, desde el punto de vista cuantitativo también nos parece interesante considerar la tendencia de cada base verbal a estar vinculada a un número mayor o menor de partículas. A este respecto, diremos que de los datos expuestos en la Tabla 2 se desprende una tendencia de los núcleos verbales a asociarse, en la gran mayoría de los casos, a una sola partícula, a diferencia por ejemplo del verbo andare, que en el corpus que nos ocupa está acompañado por 6 partículas distintas.

\begin{tabular}{|l|l|}
\hline $1 \mathrm{~V}$ con $6 \mathrm{P}$ & andare \\
\hline $2 \mathrm{~V}$ con $5 \mathrm{P}$ & tirare, mettere \\
\hline $3 \mathrm{~V} \operatorname{con} 3 \mathrm{P}$ & mandare, buttare, venire \\
\hline $6 \mathrm{~V}$ con $2 \mathrm{P}$ & andarsene, guardarsi, essere, portare, portarsi, passare \\
\hline $27 \mathrm{~V}$ con $1 \mathrm{P}$ & $\begin{array}{l}\text { rivolere, tirarsi, sgusciare, uscire, dare, starci, levare, vedersi, trovarsi, } \\
\text { ricacciare, venirsene, tagliare, circolare, scrutare, rimanere, scappare, } \\
\text { tenere, ritornare, fuggire, buttarsi, spazzare, trascinare, riportare, } \\
\text { entrare, schiodare, cacciare, stare. }\end{array}$ \\
\hline
\end{tabular}

Tabla 2. Combinación entre V y P

Además, al comparar los datos expuestos en la Tabla 1 con los recopilados en la 2, cabe subrayar que existe una correspondencia entre la frecuencia de uso de los núcleos verbales y el número de partículas con las que se combinan. De hecho, las bases verbales que se emplean con mayor frecuencia son, al mismo tiempo, las que se asocian a un número mayor de partículas, en la línea de lo que ya se ha observado en estudios anteriores (Iacobini 2008: 107-108). 


\section{Análisis del corpus paralelo: influencias tipológicas y técnicas de traducción}

Como ya hemos adelantado, desde el punto de vista tipológico, el español posee un número de VS muy bajo, con un comportamiento menos homogéneo y características menos destacables con respecto a los VS italianos (Calvo 2008: 63). Así pues, ante esta discrepancia entre el sistema italiano y el español, consideramos interesante analizar de qué manera suele responder el castellano (en cuanto lengua meta de un proceso traductor) frente al estímulo de esta categoría verbal italiana, es decir, ante un mismo esquema sintáctico ${ }^{13}$.

Del análisis del corpus paralelo se hace patente que, en la gran mayoría de los casos $(61,2 \%)$, el español suele optar por el empleo de verbos que algunos autores definen como "monorematici" (Simone 1997: 168; Cerruti 2008: 193; de ahora en adelante VM), esto es, por verbos que no están acompañados por ninguna partícula preposicional y/o adverbial ${ }^{14}$ :

TO (4): Nelle gite, noi con le nostre
scarpe chiodate, grosse, dure e pesanti
come il piombo, calzettoni di lana e pas-
samontagna, occhiali da ghiacciaio sulla
fronte, col sole che batteva a picco sulla
nostra testa in sudore, guardavamo con
invidia "i negri" che andavan su leggeri
in scarpette da tennis, o sedevano a man-
giar la panna ai tavolini degli châlet.

VS: andare su (literalmente 'ir arriba') 'subir'.
TM (19): Durante las excursiones, nosotros, con nuestros zapatos de clavos duros y pesados como el plomo, medias de lana, pasamontañas, gafas de nieve sobre la frente, y el sol cayendo de plano sobre nuestras sudorosas cabezas, mirábamos con envidia a los "palurdos" que subían, ligeros, con zapatillas de tenis, o se sentaban a tomar nata en los chiringuitos.

\begin{tabular}{|l|l|}
\hline $\begin{array}{l}\text { TO (14): "Hai visto che le Immobiliari } \\
\text { sono andate giù" }\end{array}$ & $\begin{array}{l}\text { TM (30): "Ya has visto que las } \\
\text { Inmobiliarias han bajado" }\end{array}$ \\
\hline
\end{tabular}

13. Para ser más exactos, el esquema sintáctico no es siempre exactamente el mismo, ya que el conjunto de la categoría de los VS constituye un continuum, cuyos límites coinciden con los límites de las combinaciones V+Adv. y V+SP; por ello, en ocasiones, los casos menos prototípicos de VS pueden considerarse como pertenecientes a estas otras categorías.

14. Cada tabla consta de un ejemplo paralelo, es decir, una frase del TO que contiene un VS, debidamente alineada con su correspondiente traducción al español (TM). En ambos casos, proporcionamos entre paréntesis el número de página de las ediciones de referencia. A continuación, se indica el VS en su forma infinitiva, junto con una posible traducción literal al castellano y una glosa explicativa. 
El análisis contrastivo nos permite llevar a cabo consideraciones de carácter tipológico, que subrayan algunas diferencias interesantes entre la expresión del movimiento del italiano y del español ${ }^{15}$. Al analizar la estructura del marco eventivo mediante un enfoque de tipo cognitivo (Talmy 1985: 60-61), notamos que, al igual que en los dos ejemplos que acabamos de traer a colación, algunos VS de nuestro corpus italiano (TO) están constituidos por un V de movimiento genérico (andare) -que vehicula el componente MOVIMIENTO- y una P (su, giù) -que, por su parte, se ocupa de señalar el TRAYECTO, determinando, por consiguiente, el movimiento en su globalidad-. Ahora bien, en el caso del español observamos que un mismo núcleo verbal (subir, bajar) engloba los dos componentes: MOVIMIENTO+TRAYECTO.

Por otra parte, en el seno de nuestro corpus también hallamos ejemplos que nos permiten recordar otra característica de los VS italianos desde el punto de vista aspectual. Observemos, en primer lugar, el siguiente ejemplo.

\begin{tabular}{|l|l|}
\hline $\begin{array}{l}\text { TO (40): La donna allora tirò fuori dal } \\
\text { suo pastrano una chitarra, e cominciò a } \\
\text { suonare. }\end{array}$ & $\begin{array}{l}\text { TM (61): Entonces la mujer sacó } \\
\text { una guitarra de debajo de su abrigo y } \\
\text { comenzó a tocar. }\end{array}$ \\
\hline VS: tirare fuori (lit. 'tirar fuera/lejos') 'sacar'. \\
\hline
\end{tabular}

De acuerdo con Iacobini y Masini (2007: 173-174) la partícula fuori se asocia aquí a un verbo genérico (tirare) y además de señalar el componente TRAYECTO también ejerce de indicador del telos (el punto final de una escena de movimiento), por lo que puede considerarse una partícula télica ${ }^{16}$. Ahora bien, nuestro corpus paralelo nos muestra que en la traducción de los VS al español esto no ocurre: el VM español utilizado en la traducción sigue siendo télico ${ }^{17}$, sin necesitar P alguna para señalar el telos.

Así pues, en línea con lo ya observado por Slobin (1997: 438) en un estudio contrastivo de novelas en diferentes lenguas origen y sus traducciones, en el que se destacan tendencias divergentes en lo que atañe a la forma de

15. Excepto en tres ocasiones puntuales (no relevantes desde un punto de vista cuantitativo debido a las limitaciones del corpus considerado), todas las ocurrencias analizadas constituyen verbos de movimiento. Las observaciones que vamos a proporcionar a continuación no se refieren a la totalidad de verbos de movimiento que existen en italiano con respecto a los del español, sino se ceñirán a subrayar algunas características de los VS italianos, tomándolos como punto de partida. De hecho, dentro de los sistemas lingüísticos italiano y español, numerosos verbos de movimiento cuentan con una estructura del marco eventivo de características similares.

16. De hecho, en este contexto, si eliminásemos dicha $\mathrm{P}$, el V genérico por sí mismo no tendría un valor télico.

17. Agradecemos a María Enriqueta Pérez Vázquez esta observación. 
expresar las escenas de movimiento en español y en inglés (entre otros idiomas), nos hallamos de acuerdo en que las diferencias tipológicas de este tipo acaban influyendo considerablemente en el proceso y en el resultado de la traducción. En lo que respecta a nuestro corpus, en los casos en los que un VS italiano se traduce por un VM español nos enfrentamos, pues, a traducciones constreñidas por el código lingüístico de llegada, ya que, tal como hemos señalado, subyacen unas características tipológicas determinadas, propias de la lengua meta. Por esta razón, opinamos que en estos casos no se ha hecho uso de técnicas de traducción propiamente dichas ${ }^{18}$.

Por otro lado, el 21,7\% de los VS italianos que figuran en el corpus objeto de análisis se ha traducido por la secuencia V+SP.

\begin{tabular}{|l|l|}
\hline $\begin{array}{l}\text { TO (102): "Mentre la sua famiglia era } \\
\text { dentro, lui se ne andava con le ragazze } \\
\text { a skiare!" }\end{array}$ & $\begin{array}{l}\text { TM (130): “¡Mientras su familia estaba } \\
\text { en prisión él se iba con las chicas a } \\
\text { esquiar!" }\end{array}$ \\
\hline VS: essere dentro (lit. 'estar dentro') 'estar en la cárcel'. \\
\hline
\end{tabular}

Como se puede observar del ejemplo, se trata de una solución traductora que se utiliza con frecuencia cuando en el TO se da un VS con semántica no composicional: en el contexto de uso, era dentro (lit. 'estaba dentro' estaba en la cárcel) es inmediatamente reconocible por parte de un hablante nativo de italiano, mientras que en español se precisa una suerte de explicitación del lugar del que se está hablando, ya que una traducción literal como estaba dentro resultaría del todo insuficiente y opaca. Una vez más, atendiendo al enfoque teórico en el que nos apoyamos, en estos casos tampoco es posible hablar de técnica de traducción, ya que se supedita la traducción a las restricciones del TM. Por el contrario, en otras ocasiones, cuando se recurre a la secuencia $\mathrm{V}+\mathrm{SP}$, se puede acabar empleando la técnica de traducción de la amplificación, por la que se introduce algún tipo de información que no está expresada de manera explícita en el TO, sin que sea estrictamente necesario hacerlo:

18. En nuestro análisis traductológico, en lo que se refiere al concepto de técnica de traducción, hemos adoptado el enfoque discursivo y funcional propuesto por Hurtado (2001: 264-271) según el cual, en sentido estricto, toda traducción "obligada" por el código lingüístico de llegada -al no constituir una elección entre diferentes opciones traductoras- no debe considerarse una técnica de traducción. Además, todas las técnicas de traducción que se mencionan en este trabajo, atienden a la clasificación propuesta por la misma Hurtado. 
TO (42): "Ma son maglie buone! -dicevamia madre.- Sono di Neuberg! Non vuoi mica che le butti via!".
TM (63): “Pero si son jerséis buenos! decía mi madre-. ¡Son de Neuberg! ¡No querrás que los tire a la basura!".

VS: buttare via (lit. 'tirar fuera/lejos') 'echar, tirar'.

De hecho, aquí, la referencia directa a la basura es totalmente optativa, al poderse obviar sin por ello alterar el significado global de la oración.

Otra tendencia que parece relativamente frecuente $(5,6 \%$ de las ocurrencias), a la hora de abordar la traducción de VS italianos al español, es el empleo de perífrasis verbales. En particular, entre ellas se hace patente un uso relativamente frecuente del auxiliar hacer seguido por infinitivo.

TO (60): "Mi pare che quel sempio di Terni li ha messi su contro di me".
TM (83): "Me parece que ese tonto de Terni les ha hecho ponerse contra mí".

VS: mettere su (lit. 'meter arriba') 'convencer a adoptar una actitud hostil [hacia el padre]'.

TO (88): Quanto a mia madre, lei aveva un'indole ottimista, e aspettava qualche bel colpo di scena. Aspettava che qualcuno un giorno, in qualche modo, "buttasse giù" Mussolini.
TM (115): En cuanto a mi madre, era optimista por naturaleza, y esperaba algún buen golpe de mano. Confiaba en que un día alguien "hiciese" caer a Mussolini de alguna forma.

VS: buttare giù (lit. 'tirar abajo') 'destituir [a Mussolini de su cargo]'.

TO (88): Mia madre usciva, la mattina, TM (115): Y salía por la mañana dicendo: "Vado a vedere se il fascismo è diciendo: "Voy a ver si el fascismo está sempre in piedi. Vado a vedere se hanno buttato giù Mussolini". todavía en pie. Voy a ver si han hecho caer a Mussolini".

VS: buttare giù (lit. 'tirar abajo') 'destituir [a Mussolini de su cargo]'.

TO (204): Era molto in uso fra noi fare l'autocritica, un tempo, negli anni del dopoguerra: cioè dopo aver commesso errori, analizzarli e sezionarli a voce alta. Intrecciavamo errori su errori; e l'autocritica veniva a sovrapporsi agli errori, si intrecciava e si confondeva con quegli stessi errori, al modo come la musica si confonde con le parole d'un'opera, ne oscura il senso e se le porta via nel suo ritmo di gloria.
TM (248): En una época, en los años de la posguerra, era costumbre entre nosotros hacer la autocrítica, que consistía en analizar y en diseccionar en voz alta los errores que habíamos cometido. Entrelazábamos unos errores con otros y la autocrítica se superponía, se entrelazaba y se confundía con ellos, del mismo modo que la música se confunde con la letra de una ópera, oscurece su sentido y la hace desaparecer en su ritmo glorioso.

VS: portarsi via (lit. 'llevarse fuera/lejos') 'llevarse [algo]'. 
Es interesante señalar que, en las tres primeras ocasiones, la perífrasis hacer+infinitivo es utilizada para la traducción de VS idiomáticos, con significado no composicional. Ahora bien, puesto que la traductora elige estas equivalencias entre otras opciones posibles ${ }^{19}$, cabe considerar estos casos como ejemplos de transposición, dado el cambio de categoría gramatical que esta técnica implica (pues el VS italiano, pasa a ser una perífrasis de hacer+infinitivo). Por otro lado, el último ejemplo de empleo de esta perífrasis concierne a un VS con significado composicional, portare via, que aquí se traduce mediante la técnica de la particularización (en lugar de optar por una solución más literal como se la lleva).

Otra solución traductora que figura en el corpus español con relativa frecuencia $(4,8 \%)$ es la asociación de un verbo seguido por un adverbio (V+Adv.).

\begin{tabular}{|l|l|}
\hline TO (192): "Quand'ero in galera", diceva & TM (234): "Cuando estaba en la cárcel", \\
spesso. In galera, diceva, s'era sentita & $\begin{array}{l}\text { decía a menudo. En la cárcel, decía, se } \\
\text { molto a suo agio, finalmente a posto, in } \\
\text { había sentido muy a sus anchas, por fin } \\
\text { pace con se stessa, libera di complessi } \\
\text { bien y en paz consigo misma, libre de } \\
\text { e d'inibizioni. Aveva fatto amicizia con } \\
\text { delle ragazze jugoslave, che erano den- } \\
\text { tro per motivi politici, e anche con delle e de inhibiciones. Se había } \\
\text { hecho amiga de unas chicas yugoslavas } \\
\text { que estaban allí por motivos políticos, y } \\
\text { también de unas presas comunes. }\end{array}$ \\
\hline \multicolumn{2}{|l|}{ VS: essere dentro (lit. 'estar dentro') 'estar en la cárcel'. } \\
\hline
\end{tabular}

El empleo en español de esta combinación parece responder a exigencias de tipo contextual, pues depende de dónde se inserte el constructo. A falta de un VS equivalente, en esta ocasión la combinación V+Adv. permite evitar la repetición del sintagma en la cárcel, ya mencionado en el seno del mismo párrafo, mediante un mecanismo anafórico ${ }^{20}$. La diferencia entre las dos lenguas estriba en que en italiano se observa un proceso metonímico del tipo LA PARTE POR EL TODO por el que el VS (essere dentro) se ha gramaticalizado y, por ello, la $\mathrm{P}$ (dentro) del constructo resulta anclada siempre al mismo referente (la cárcel); por otro lado, en español el adverbio allí constituye una mera referencia anafórica, de carácter variable, contextual.

En otros casos, el uso de dicha combinación parece poner de manifiesto tendencias divergentes en la expresión de la deixis espacial:

19. A modo de ejemplo, proponemos como alternativas: 'les ha puesto en mi contra', 'tumbara', 'ha tumbado', respectivamente.

20. A este respecto, señalamos que este problema no se da en italiano, precisamente gracias a la riqueza de su sistema lingüístico en este campo léxico, ya que dispone de un VS para expresar ese mismo significado (essere dentro, 'estar en la cárcel'). 
TO (209): Gabriele, mio marito, mi scri- TM (253-254): Gabriele, mi marido, me veva da Roma che mi sbrigassi a venir escribía desde Roma diciéndome que me giù coi bambini.

diese prisa en ir allí con los niños.

VS: venire giù (lit. 'venir abajo') 'ir [de una ciudad situada en el norte de Italia (Turín) a una ciudad ubicada en el centro de Italia (Roma)]'.

Aquí, el VS venire giù (lit. 'venir abajo', bajar) es empleado para describir el desplazamiento de Natalia desde la ciudad de Turín (en el norte de Italia) a Roma (en la zona central de la península), la ciudad en la que se encuentra su marido. En este caso, al margen de la conocida discrepancia entre el uso de andare/venire e ir/venir en las dos lenguas en cuestión, en el VS italiano el TRAYECTO señalado por giù (que designa un movimiento en el espacio de norte a sur), no se vehicula en español de la misma forma, pues la referencia directa al desplazamiento hacia el sur queda de alguna manera implícita, al emplear el adverbio de lugar allí. Si bien en el español (al menos peninsular) en intercambios informales se dan casos de uso del verbo bajar ${ }^{21}$ o subir para designar desplazamiento físico hacia el sur o norte con respecto al hablante (o de una localidad situada a mayor altura a otra más baja, o de una de mayor entidad a otra menor), el análisis del corpus apunta a que parece más frecuente recurrir, en su lugar, a una referencia deíctica como allí (que se justifica por el contexto, pues retoma el antecedente Roma), del todo parecida a la del ejemplo anterior. Al existir una opción alternativa frente a la que figura en el TM, no se trata de una solución obligada por la lengua de llegada, sino más bien de una elección por parte de la traductora ${ }^{22}$.

Por último, las restantes soluciones traductoras que se hallan en el corpus $(6,7 \%$ de las ocurrencias) representan un conjunto de difícil clasificación, puesto que no son homogéneas ni catalogables bajo un mismo grupo y cuentan, en la mayoría de las ocasiones, con una única ocurrencia a lo largo de todo el texto paralelo considerado. Por ello, en dichos casos, debido a las limitaciones del mismo corpus, no nos ha sido posible delinear patrones lingüísticos relevantes desde un punto de vista cuantitativo.

A modo de resumen, señalamos a continuación una tabla para reflejar en términos porcentuales las tendencias traductoras que nuestro estudio ha destacado.

21. Esta acepción del verbo bajar ha dado lugar a expresiones coloquiales como bajar(se) al moro.

22. Si nos ceñimos a la traducción del VS, podemos considerar este caso como un ejemplo de generalización, una técnica por la que el desplazamiento de norte a sur vehiculado por giù en el TO, pasa a designar con allí un simple movimiento. 


\begin{tabular}{|c|c|c|}
\hline ITALIANO & ESPANOL & \% OCURRENCIAS \\
\hline VS & VM & $61,2 \%$ \\
\hline VS & V+SP & $21,7 \%$ \\
\hline VS & Perífrasis verbal & $5,6 \%$ \\
\hline VS & V+Adv. & $4,8 \%$ \\
\hline VS & Soluciones puntuales & $6,7 \%$ \\
\hline
\end{tabular}

\section{Los VS como problema de traducción}

Desde el punto de vista traductológico cabe destacar, en primer lugar, que el TM de nuestro corpus paralelo constituye, en términos generales, una traducción de buena calidad. De hecho, a pesar de la gran complejidad del estilo y del tono general propio del TO, el TM se muestra fiel al método interpretativo-comunicativo ${ }^{23}$ adoptado en el proceso de traducción. Sin embargo, no es posible asegurar lo mismo en lo que atañe a la traducción de los constructos sintagmáticos verbales, pues desde este punto de vista en nuestro análisis hemos hallado numerosos y diversos errores de traducción ${ }^{24}$.

Para empezar, se observa una tendencia relativamente frecuente a traducir de forma errónea el significado de aquellos VS que presentan una semántica no composicional. La opacidad semántica de algunos VS idiomáticos parece conllevar problemas de comprensión para la profesional que se enfrenta a ellos. De hecho, el italiano constituye la L2 de la traductora y, por consiguiente, parece lógico suponer que en este idioma su percepción lingüística en cuanto a los significados idiomáticos de estas estructuras es inferior a la de un nativo; además, como ya hemos señalado, tanto los diccionarios como las gramáticas disponibles hoy en día para quienes estudian el italiano no abordan la cuestión de los VS de manera exhaustiva y ordenada, por lo que ante una duda concerniente a su significado el traductor difícilmente podrá recuperar la información que precisa para llevar a cabo su labor; por último, no

23. Para una clasificación de los posibles métodos de traducción, hacemos referencia a la propuesta de Hurtado (2001: 252), quien define el método interpretativo-comunicativo como "método traductor que se centra en la comprensión y reexpresión del sentido del texto original conservando la traducción la misma finalidad que el original y produciendo el mismo efecto en el destinatario; se mantiene la función y el género textual".

24. Adoptamos la concepción funcional de error de traducción que se detalla en Hurtado (2001: 302-308), por la que un error "solo puede analizarse desde una perspectiva textual, contextual y funcional que considere el elemento en cuestión en relación con el conjunto del texto, con el contexto en que se efectúa la traducción [...] con la finalidad de la traducción y el método elegido, con el tipo y modalidad de traducción de que se trate [...]" (2001: 303). 
cabe olvidar que los VS pertenecen a una categoría verbal abierta, en continua expansión, y que en esta novela hay incluso casos de VS que parecen responder al idiolecto de algún personaje o al contexto dialectal. En otras palabras, desde este punto de vista, los VS constituirían un verdadero problema de traducción, de carácter tanto lingüístico como instrumental ${ }^{25}$.

Por todo ello, la traductora trata de solucionar esta cuestión facilitando una interpretación personal del constructo verbal, que está basada en el contexto oracional en el que se enmarca, lo que a su vez, en ocasiones, da lugar a dos tipos de errores de traducción: el "falso sentido" (FS) y el "no mismo sentido" (NMS) (Agost \& Monzó 2001: 18-19). En cuanto al primero, en las ocurrencias que exponemos a continuación, se observa que la traductora, a causa de la opacidad semántica del VS, cae en un FS por no haber sabido captar el significado idiomático de la construcción verbal:

\begin{tabular}{|l|l|}
\hline $\begin{array}{l}\text { TO (8): "Ho una tosse che mi strozzo", } \\
\text { diceva dopo un poco a mio padre, che } \\
\text { sempre tirava avanti e non si voltava. }\end{array}$ & $\begin{array}{l}\text { TM (23): "Tengo una tos que me ahogo", } \\
\text { decía poco después a mi padre, que iba } \\
\text { siempre delante y no se volvía. }\end{array}$ \\
\hline VS: tirare avanti (lit. 'tirar/ir adelante') 'seguir caminando'.
\end{tabular}

\begin{tabular}{|c|c|}
\hline $\begin{array}{l}\text { TO (79): Chiese la mano di mia sorella } \\
\text { in un attimo; e poi però rimase ancora } \\
\text { un pezzo in poltrona nel nostro salotto, } \\
\text { trastullandosi con la sua barba, e raccon- } \\
\text { tando di sé: come aveva tirato su la sua } \\
\text { fabbrica, con pochi soldi, e come aveva } \\
\text { educato tutti i suoi figli, e come leggeva } \\
\text { ogni sera, prima d'addormentarsi, la } \\
\text { Bibbia. }\end{array}$ & $\begin{array}{l}\text { TM (105): Pidió la mano de mi hermana } \\
\text { en un segundo, pero después se quedó } \\
\text { sentado durante bastante rato en un } \\
\text { sillón de nuestro salón, jugueteando con } \\
\text { su barba y contándonos cosas de él. Nos } \\
\text { habló de cómo había sacado adelante su } \\
\text { fábrica con muy poco dinero, de cómo } \\
\text { había educado a todos sus hijos y de } \\
\text { cómo leía la Biblia todas las noches antes } \\
\text { de dormirse. }\end{array}$ \\
\hline
\end{tabular}

TO (212): "Forse non li avevi cercati bene Beppino!" -disse mia madre-. "Forse li dovevi cercare ancora!"

- "Macché! Sempia che non sei altro! Non era mica una cosa semplice! Sei subito pronta a buttarmi giù. Ma guarda che asina che sei!".
TM (257): “¡Tal vez no los buscaste bien, Beppino! -dijo mi madre-. ¡Tal vez los deberías haber seguido buscando!"

- "¡Tonta, que no eres otra cosa! ¡No era tan sencillo! Estás siempre dispuesta a dejarme mal. ¡Pero mira que eres borrica!".

VS: buttare giù (lit. 'tirar abajo') 'desanimar [a alguien]'.

25. Atendemos aquí a la clasificación de problemas de traducción de Hurtado (2001: 288). 
En particular, en el primer ejemplo, tirare avanti (lit. 'tirar/ir adelante') ha de considerarse idiomático pues, lejos de tener sentido composicional, significa 'seguir caminando', un matiz de significado que la traductora no ha comprendido, y que nada tiene que ver con el hecho de que el padre de Natalia durante las excursiones a la montaña fuera siempre delante de su mujer, tal y como se deduce de la traducción al español.

Por otro lado, en el segundo, se produce un FS más, ya que tirare su (lit. 'tirar arriba') una fábrica no se corresponde con "sacarla adelante", sino que implica el hecho de montar la empresa desde cero.

Finalmente, en el tercero, nos enfrentamos con otro VS idiomático, buttare giù (lit. 'tirar abajo'). Aquí, a partir del contexto en el que la expresión se inserta la traductora ha comprendido que se trata de un VS con acepción negativa, pues el padre y la madre de Natalia están discutiendo como de costumbre, reprochándose mutuamente algunas cosas. A pesar de que el verbo empleado en la traducción encaja muy bien en el contexto de uso, se trata una vez más de un FS, pues el VS en cuestión no significa 'dejar mal a alguien' (frente a los demás) sino más bien 'desanimar a alguien'.

La idiomaticidad de las construcciones sintagmáticas ha acarreado problemas de comprensión en una ocasión más, en la que se observa otro tipo de error traductor, el NMS.

\begin{tabular}{|l|l|}
\hline TO (34): Si stufava però presto; s'im- & TM (53): Pero enseguida se enfurruñaba \\
bronciava, si stizziva con me e con & $\begin{array}{l}\text { y se aburría. Entonces se enfadaba con- } \\
\text { Lucio, che le stavamo intorno perché } \\
\text { migo y con Lucio, que estábamos a su } \\
\text { prima ci aveva promesso passeggiate e e } \\
\text { caramelle [...]. }\end{array}$ \\
$\begin{array}{l}\text { alrededor, porque antes nos había pro- } \\
\text { metido llevarnos de paseo y darnos cara- } \\
\text { melos [...]. }\end{array}$ \\
\hline VS: stare intorno (lit. 'estar alrededor') 'estar encima [de alguien]'. \\
\hline
\end{tabular}

De hecho, tal y como se observa en este ejemplo, stare intorno (lit. 'estar alrededor') tiene aquí un valor idiomático, puesto que significa 'estar encima de alguien' con el objeto de influenciar su comportamiento e insistir para que esa persona lleve a cabo una acción determinada (llevar a los niños a dar un paseo y comprarles caramelos). Al no haberse comprendido la idiomaticidad de la construcción, en el TM se observa una traducción literal que resulta extraña en castellano (ya que parece poco natural) y fuera de lugar en este contexto.

En definitiva, los errores que hemos considerado hasta el momento, si bien no afectan al texto en su globalidad, tienen una repercusión de tipo lingüístico, pues no reproducen de forma fiel el mensaje expresado por el TO. 
Por último, en algunas ocasiones la traducción presenta un registro ${ }^{26}$ excesivamente elevado y formal con respecto al VS que encontramos en el TO. A este respecto, consideramos que, por las mismas razones aducidas anteriormente en lo que se refiere a los errores de carácter semántico, los VS pueden constituir un problema de traducción de carácter lingüístico e instrumental. La escasa consideración de este fenómeno lingüístico por parte de las fuentes lexicográficas y el hecho de que la traducción la haya llevado a cabo un hablante no nativo de italiano hacen que la correcta ubicación de los VS en el eje diafásico resulte más complicada. Por ello, en la traducción se hace un uso erróneo de la técnica de la variación. Ofrecemos a continuación tres ejemplos de errores que atañen al registro (REG).

\begin{tabular}{|c|c|}
\hline $\begin{array}{l}\text { TO (96): Quando s'era buttato nel fiume, } \\
\text { una guardia aveva tirato fuori la pistola; } \\
\text { ma un'altra guardia aveva gridato di non } \\
\text { sparare. }\end{array}$ & $\begin{array}{l}\text { TM (124): Cuando se tiró al río un poli- } \\
\text { cía desenfundó la pistola, pero otro le } \\
\text { gritó que no disparase. }\end{array}$ \\
\hline
\end{tabular}

TO (23): Come mai da quella stirpe TM (40): No sé cómo mi padre y su herdi banchieri, che erano gli antenati e i mano Cesare, que no tenían ningún senparenti di mio padre, siano usciti fuori tido de los negocios, podían proceder de mio padre e suo fratello Cesare, del tutto una estirpe de banqueros como los antedestituiti d'ogni senso degli affari, non pasados y parientes de mi padre. so.

VS: uscire fuori (lit. 'salir fuera') 'proceder'.

TO (74): "Non gli va via la febbre. TM (99): "No le desaparece la fiebre. Ho paura che abbia un versamento ¡Temo que tenga un derrame pleural! pleurico". [...]".

VS: andare via (lit. 'ir lejos/fuera') 'írsele [la fiebre], desaparecer [la fiebre]'.

26. En el seno de este trabajo, aunque para simplificar nuestro análisis hablaremos del registro de la obra sin diferenciar entre las tres variables que lo componen (campo, tenor y modo), nos referiremos principalmente al campo y al modo, por las razones que en breve se detallarán. Por otro lado, cabe añadir que al abordar el estudio traductológico se ha tenido en cuenta que, tal y como se observa en Marco (2002:73), "la relació entre text i registre no és biunívoca, ja que, en la majoria dels casos, un text conté més d'un registre. Això és especialment cert als textos literaris, on hi ha canvis constants de registre relacionats amb una o més variables". Aquí, sin embargo, cuando hablemos de registro, nos referiremos estrictamente a los apartados más informales y coloquiales de la obra, que en la mayoría de los casos se plasman bien bajo la forma del estilo directo, bien mediante el relato de la narradora, que retoma expresiones peculiares de los Levi o da cuenta de sus costumbres empleando un lenguaje marcado tanto diafásica como diamésicamente (como el mismo empleo de los VS nos confirma). 
Tal y como se observa en el primer ejemplo, el VS tirare fuori (lit. 'tirar fuera') se ha traducido por desenfundar, es decir, un VM cuya ubicación en el eje diafásico resulta sin lugar a dudas desplazada hacia un mayor nivel de formalidad frente al VS italiano. A nuestra manera de ver, la propuesta traductora de la profesional sería válida si en el TO apareciera el verbo estrarre que, aparte de ser un VM, también pertenece a un registro más elevado. En cambio, dadas las características de tirare fuori, y del contexto general en el que el parlamento se inserta, opinamos que sacar sería un verbo más adecuado para su traducción.

Asimismo, en los dos ejemplos siguientes, la traductora recurre a un VM propio de un registro más elevado con respecto al VS italiano, otorgando un carácter excesivamente formal a un discurso que, por el contrario, se presenta al lector italiano como un relato cotidiano, muy cercano a la oralidad y caracterizado por una tendencia generalizada hacia la informalidad. En estos casos, como traducciones alternativas proponemos debían haber salido y no se le va, respectivamente.

En cuanto a este segundo tipo de error, a modo de reflexión general, diremos que su repercusión ha de situarse en el plano pragmático: mediante estas equivalencias traductoras, aunque la semántica de los VS del original queda reproducida, se acaba alterando la intención de Natalia Ginzburg, pues en el TM no se reproduce el tono de informalidad que caracteriza el TO.

\section{Conclusión}

El desarrollo de la investigación ha confirmado nuestra hipótesis inicial, esto es, que la obra Lessico famigliare (y por extensión otras similares a las que estamos extendiendo nuestro estudio) representa una fuente idónea para poder hallar ejemplos de VS, siendo estos constructos particularmente productivos en textos -como el que nos ocupa- con características próximas a la oralidad.

Por otra parte, en el análisis contrastivo del corpus paralelo hemos destacado que en la mayoría de los casos ante un VS italiano la traducción al español recurre a un VM. Tal y como hemos observado, dicha solución traductora parece responder a diferencias de carácter tipológico entre los dos códigos lingüísticos, esto es, a una diferente distribución de los componentes del marco eventivo de los verbos de movimiento del español frente al italiano. Las demás opciones traductoras, menos significativas desde el punto de vista cuantitativo debido a las limitaciones del corpus considerado, presentan una tendencia bipartita: en algunos casos, son elecciones constreñidas por la lengua de llegada (a menudo debido a discrepancias de carácter tipológico); en 
otros, en cambio, han de considerarse como técnicas de traducción propiamente dichas, pues han sido elegidas por la traductora entre varias opciones posibles.

Hemos señalado, asimismo, la propensión en la traducción analizada a cometer errores en lo que afecta a la semántica de los VS no composicionales y a la identificación del registro de algunos constructos. A este respecto, hemos intentado señalar las posibles causas de los errores en cuestión, así como su repercusión e incidencia.

Conscientes de las limitaciones de este estudio de caso, en la actualidad nos hallamos en fase de ampliación de nuestra base de datos de textos paralelos, con el objeto de comprobar si los patrones lingüísticos y traductológicos aquí delineados corresponden a conductas generalizadas a la hora de abordar la traducción del italiano al español de los constructos sintagmáticos verbales. En otras palabras, observaremos si las tendencias que hemos destacado se vuelven a dar de forma sistemática en textos diferentes, correspondientes a traductores distintos.

\section{Referencias bibliográficas}

Agost, Rosa \& Esther Monzó. (2001) Teoria i pràctica de la traducció general, Espanyol-català. Castellón: Publicacions de la Universitat Jaume I.

ANTELMI, Donella. (2002) "Il verbo senza significato: possibilità di slittamento del contenuto lessicale su elementi di tipo nominale." Rivista italiana di linguistica e di dialettologia 4, pp. 97-117.

BARANI, Valeria. (1990) "Il 'latino' polifonico della famiglia Levi nel "Lessico famigliare" di Natalia Ginzburg." Otto/Novecento 6, pp. 147-157.

Calvo Rigual, Cesáreo. (2005) "El Galateo di Giovanni della Casa y sus traducciones, fuente de contrastes léxicos." En: Claveria, Glòria \& Cristina Buenafuentes (eds.) 2005. Germà Colón: les llengües romàniques juntes $i$ contrastades. Bellaterra (Barcelona): Universitat Autònoma de Barcelona, pp. 27-49.

Calvo Rigual, Cesáreo. (2008) "I verbi sintagmatici italiani, con appunti contrastivi con lo spagnolo e il catalano." En: González Royo, Carmen \& Pedro Mogorrón Huerta (eds.) 2008. Estudios y Análisis de Fraseología Contrastiva: Lexicografía y Traducción. Alicante: Universidad de Alicante, pp. 47-66.

CAlvo RiguAL, Cesáreo. (2010) "Trattamento nella lessicografia monolingue (italiana) e bilingue (italiano-spagnolo e catalano) dei verbi sintagmatici: panorama attuale e proposte future." En: Iliescu, Maria; Heidi Siller-Runggaldier \& Paul Danler (eds.) 2010. Actes du XXVe Congrès International de Linguistique et Philologie Romanes. Berlín \& Nueva York: Walter de Gruyter, vol. 7, pp. 375-383. 
Carrera Díaz, Manuel. (1984) Curso de lengua italiana. Parte teórica. Barcelona: Ariel.

Cerruti, Massimo. (2008) "Verbi sintagmatici e sinonimi monorematici nell'italiano parlato. La dimensione diafasica, diatopica, diastratica." En: Cini, Monica (ed.) 2008. I verbi sintagmatici in italiano e nelle varietà dialettali - Stato dell'arte e prospettive di ricerca. Atti delle giornate di studio. Torino 19-20 febbraio 2007. Fráncfort del Meno: Peter Lang, pp. 193-208.

GINZBURG, Natalia. (1963) Lessico famigliare. Turín: Einaudi.

GinzBurg, Natalia (traducción de Mercedes Corral). (1963) Léxico familiar. Barcelona: Trieste, 1989. Ed. citada: Barcelona: Lumen, 2007.

HARTMAnN, Reinhard Rudolf Karl. (1980) Contrastive Textology. Comparative Discourse Analysis in Applied Linguistics. Heidelberg: Julius Groos.

HuRTAdo Albir, Amparo. (2001) Traducción y traductología. Introducción a la traductología. Madrid: Cátedra.

IACOBINI, Claudio. (2008) "Presenza e uso dei verbi sintagmatici nel parlato dell'italiano." En: Cini, Monica (ed.) 2008. I verbi sintagmatici in italiano e nelle varietà dialettali - Stato dell'arte e prospettive di ricerca. Atti delle giornate di studio. Torino 19-20 febbraio 2007. Fráncfort del Meno: Peter Lang, pp. 103-119.

IACOBINI, Claudio \& Francesca Masini. (2007) "Verb-particle Constructions and Prefixed Verbs in Italian: Typology, Diachrony and Semantics." En: Booij, Geert; Luca Ducceschi; Bernard Fradin; Emiliano Guevara; Angela Ralli $\&$ Sergio Scalise (eds.) 2007. On-line Proceedings of the Fifth Mediterranean Morphology Meeting (MMM5). Fréjus 15-18 September 2005. Bolonia: Universidad de Bolonia. Versión electrónica: <http://www3.lingue.unibo.it/ mmm2/wp-content/uploads/2012/09/157-184-Iacobini-Masini.pdf>

JEŽEK, Elisabett \& Francesca Strik Lievers. (2010) "Verbi sintagmatici in italiano antico e moderno: un'analisi corpus-based." En: Iliescu, Maria; Heidi SillerRunggaldier \& Paul Danler (eds.) 2010. Actes du XXVe Congrès International de Linguistique et Philologie Romanes. Berlín \& Nueva York: Walter de Gruyter, pp. 445-454.

MAgRini, Giacomo. (1996) "Lessico famigliare di Natalia Ginzburg." En: Asor Rosa, Alberto (ed.) 1996. Letteratura italiana. Le Opere, 4 (2). Turín: Einaudi, pp. 771-810.

MArco, Josep. (2002) El fil d'Ariadna: anàlisi estilística i traducció literària. Vic (Barcelona): Eumo.

MASINI, Francesca. (2005) "Multi-Word Expressions between Syntax and the Lexicon: The Case of Italian Verb-Particle Constructions." SKY Journal of Linguistics18, pp. 145-173.

Masini, Francesca. (2008) "Verbi sintagmatici e ordine delle parole." En: Cini, Monica (ed.) 2008. I verbi sintagmatici in italiano e nelle varietà dialettali 
- Stato dell'arte e prospettive di ricerca. Atti delle giornate di studio, Torino 19-20 febbraio 2007. Fráncfort del Meno: Peter Lang, pp. 83-102.

MASINI, Francesca. (2012) Parole sintagmatiche in italiano. Cesena \& Roma: Caissa Italia.

Mioni, Alberto. (1983) "Italiano tendenziale: osservazioni su alcuni aspetti della standardizzazione." En: Benincà, Paola; Guglielmo Cinque; Tullio De Mauro \& Nigel Vincent (eds.) 1983. Scritti linguistici in onore di Giovan Battista Pellegrini. Pisa: Pacini, pp. 495-517.

SimONE, Raffaele. (1997) "Esistono verbi sintagmatici in italiano?" En: De Mauro, Tullio \& Vincenzo Lo Cascio (eds.) 1997. Lessico e grammatica. Teorie linguistiche e applicazioni lessicografiche. Atti del Convegno interannuale della Società di Linguistica Italiana. Madrid, 21-25 febrero de 1995. Roma: Bulzoni, pp. 155-170.

SCHWARZE, Christoph. (1985) "Uscire' e 'andare fuori': struttura sintattica e semantica lessicale." En: Franchi de Bellis, Annalisa \& Leonardo Maria Savoia (eds.) 1985. Sintassi e morfologia della lingua italiana d'uso. Teorie e applicazioni descrittive. Atti del XVII Congresso internazionale di studi. Urbino, 11-13 de septiembre de 1983. Roma: Bulzoni, pp. 355-371.

Slobin, Dan Isaac. (1997) "Mind, Code and Text." En: Bybee, Joan; John Haiman \& Sandra A. Thompson (eds.) 1997. Essays on Language Function and Language Type. Ámsterdam \& Filadelfia: John Benjamins, pp. 437-467.

TALMY, Leonard. (1985) "Lexicalization patterns semantic structure in lexical forms." En: Shopen, Timothy (ed.) 1985. Language Typology and Syntactic Description, Vol. III. Grammatical Categories and the Lexicon. Cambridge: Cambridge University Press, pp. 57-149.

VALERO GARCÉS, Carmen. (1995) Apuntes sobre traducción literaria y análisis contrastivo de textos literarios. Alcalá de Henares: Publicaciones de la Universidad de Alcalá.

Venier, Federica. (1996) "I verbi sintagmatici." En: Blumenthal, Peter \& Christoph Schwarze (eds.) 1996. Lexikalische Analyse Romanischer Sprachen. Tubinga: Max Niemeyer, pp. 149-156.

WANDRUSZKA, Mario. (1969) Sprachen-Vergleichbar und Unvergleichlich. Múnich: Piper.

WANDRUSZKA, Mario. (1971) Interlinguistik:Umrisseeinerneuen Sprachwissenschaft. Múnich: Piper. 


\section{NOTA BIOGRÁFICA / BIONOTE}

ANDREA ARTUSI es licenciado en Lingue e Letterature Straniere por la Università degli Studi di Verona y en Traducción e Interpretación por la Universidad de Valencia. En 2012, adquirió el título de Máster en Traducción Creativa y Humanística (Universidad de Valencia). Es miembro actual del grupo de investigación COMETVAL, con el proyecto "Análisis léxico y discursivo de corpus paralelos y comparables (español-inglés-francés) de páginas electrónicas de promoción turística", perteneciente al IULMA (Instituto Interuniversitario de Lenguas Modernas Aplicadas). En la actualidad, está cursando sus estudios de doctorado en la Universidad de Valencia, en el ámbito de la traductología.

ANDREA ARTUSI holds a B. A. in Foreign Languages and Literatures (Spanish and English) from the University of Verona (Italy) and a B.A. in Translation and Interpreting (Spanish, English and Italian) from the University of Valencia (Spain). In 2012 he received his Master's Degree in Creative and Humanistic Translation from the same university. He is a member of the COMETVAL research group, working on the project "Lexical and discourse analysis of parallel and comparable corpora (Spanish-English-French) of web pages for tourism promotion", belonging to the IULMA (Interuniversity Institute for Applied Modern Languages). He is currently studying towards his Ph.D. at the University of Valencia, within the field of translation studies. 